\title{
ON NILSTABLE ALGEBRAS
}

\section{LOUIS A. KOKORIS}

1. Introduction. A simple commutative power-associative algebra $\mathfrak{A}$ of degree 2 over a field $\mathfrak{F}$ of characteristic not 2 has a unity element $1=u+v$ where $u$ and $v$ are orthogonal idempotents. Then $\mathfrak{A}$ may be decomposed relative to $u$ and written as $\mathfrak{U}=\mathfrak{A}_{1}+\mathfrak{U}_{12}+\mathfrak{U}_{2}$ with $\mathfrak{A}_{1}$ $=\mathfrak{A}_{u}(1)=\mathfrak{A}_{v}(0), \mathfrak{A}_{12}=\mathfrak{A}_{u}(1 / 2)=\mathfrak{A}_{v}(1 / 2)$ and $\mathfrak{A}_{2}=\mathfrak{A}_{u}(0)=\mathfrak{A}_{v}(1)$ where $x$ is in $\mathfrak{A}_{u}(\lambda)$ if and only if $x u=\lambda x$. Furthermore $\mathfrak{A}_{1}=u \mathfrak{F}+\mathfrak{S}_{1}$ and $\mathfrak{H}_{2}=v \mathfrak{F}+\mathfrak{G}_{2}$ where $\mathfrak{S}_{1}$ and $\mathfrak{G}_{2}$ are nilalgebras. It is known that $\mathfrak{A}_{1}$ and $\mathfrak{A}_{2}$ are orthogonal subalgebras of $\mathfrak{A}, \mathfrak{A}_{12}^{2} \subseteq \mathfrak{A}_{1}+\mathfrak{A}_{2}$, and $\mathfrak{A}_{12} \mathfrak{A}_{i}$ $\subseteq \mathfrak{A}_{12}+\mathfrak{A}_{3-i}$ for $i=1,2 .{ }^{1}$ Albert has defined $u$ to be a stable idempotent and $\mathfrak{A}$ to be $u$-stable in case $\mathfrak{A}_{12} \mathfrak{A}_{i} \subseteq \mathfrak{A}_{12}$ for $i=1,2$. We generalize this notion and call $u$ a nilstable idempotent and $\mathfrak{U}$ nilstable with respect to $u$ if $\mathfrak{A}_{12} \mathfrak{A}_{i} \subseteq \mathfrak{A}_{12}+\mathfrak{G}_{3-i}$ for $i=1,2$. Thus every stable idempotent is also nilstable. It is known that every commutative powerassociative algebra of degree 2 and characteristic 0 is nilstable with respect to every idempotent. ${ }^{2}$

The purpose of this note is to give the proof of the following theorem.

THEOREM 1. Let $\mathfrak{A}$ be a simple commutative power-associative algebra of degree 2 over a field $\mathfrak{F}$ whose characteristic is prime to 6. Then $\mathfrak{A}$ is a Jordan algebra if and only if $\mathfrak{A}$ is nilstable with respect to two idempotents $u, f$ such that $u \neq 1, f \neq 1, u+f \neq 1$ and such that $f$ is not of the form $f=u+w_{12}+w_{1}+w_{2}$ or $f=v+w_{12}+w_{1}+w_{2}$ with $w_{12}$ in $\mathfrak{A}_{12}, w_{1}$ in $\mathfrak{G}_{1}, w_{2}$ in $\mathfrak{G}_{2}$.

Since any Jordan algebra is stable with respect to each of its idempotents, we are only concerned with the other half of the theorem. The proof is to a large extent the proof in [8] of the result that every simple commutative power-associative algebra of degree 2 and characteristic 0 is a Jordan algebra. Since we shall lean rather heavily on [8], we shall refer the reader to that paper instead of repeating those results here.

The simple $u$-stable algebras have been determined by Albert $[3 ; 4 ; 5]$. There remains the problem of finding all algebras which 1958.

Presented to the Society April 18, 1958, received by the editors February 28,

1 The above results are given in $[1,2$, and 7$]$.

2 See [7]. 
are not $u$-stable. It is hoped that Theorem 1 will be useful in solving the intermediate problem of determining the nilstable algebras.

2. Idempotents. Let $\mathfrak{A}$ be nilstable with respect to an idempotent $u$. Then if $\mathfrak{F}$ has characteristic not 2 or 3 , all the results of $\$ 2$ of [8] are valid here. This is because the assumption of nilstability is the assumption of the conclusion of Lemma 1 of [8]. Characteristic not 2 is needed from the outset and it is necessary to divide by 3 at the end of the proof of Lemma 3.

A result needed to prove Theorem 1 is

THEOREM 2. Let $\mathfrak{A}$ be a commutative power-associative algebra of degree 2 and let $u$ be a nilstable idempotent of $\mathfrak{A}$. If $f$ is any idempotent of $\mathfrak{A}$ other than $u, v, 1$, then $f=1 / 2(1+w)$ where $w^{2}=1$ and $w=\gamma(u-v)$ $+w_{12}+w_{1}+w_{2}$ where $w_{12} \neq 0$ is in $\mathfrak{A}_{12}, w_{i}$ is in $\mathfrak{B}_{i}, i=1,2$.

If $f$ is any idempotent, then $w=2 f-1$ has the property $w^{2}=1$. Then $w=\gamma u+\delta v+w_{12}+w_{1}+w_{2}$ where $\gamma, \delta$ are in $\mathfrak{F}, w_{12}$ in $\mathfrak{A}_{12}, w_{1}$ in $\mathfrak{H}_{1}, w_{2}$ in $\mathfrak{G}_{2}$. When $w_{12}=0, w^{2}=1=\gamma^{2} u+\delta^{2} v+2 \gamma w_{1}+2 \delta w_{2}+w_{1}^{2}+w_{2}^{2}$. Consequently, $\quad \gamma^{2}=\delta^{2}=1, \quad 2 \gamma w_{1}+w_{1}^{2}=0, \quad 2 \delta w_{2}+w_{2}^{2}=0$. Since $\gamma \neq 10, \quad w_{1}^{2}$ $=-2 \gamma w_{1}$ implies $\left(-(1 / 2 \gamma) w_{1}\right)^{2}=-(1 / 2 \gamma) w_{1}$ so $-(1 / 2 \gamma) w_{1}$ is idempotent or zero. But $w_{1}$ is nilpotent so $w_{1}=0$. Similarly, $w_{2}=0$. Thus $w=\gamma u+\delta v$ where $\gamma= \pm 1, \delta= \pm 1$. It follows that $w=1,-1, u-v$, or $v-u$, and $f=1 / 2(1+w)=0,1, u$, or $v$. By hypothesis $f$ is not $0,1, u$, or $v$, so $w_{12}$ must be nonzero.

Computing $w^{2}=1$ we have $\gamma^{2} u+\delta^{2} v+(\gamma+\delta) w_{12}+w_{12}^{2}+2 w_{12}\left(w_{1}+w_{2}\right)$ $+2 \gamma w_{1}+2 \delta w_{2}+w_{1}^{2}+w_{2}^{2}=1$. Equating components in $\mathfrak{A}_{12},(\gamma+\delta) w_{12}$ $+2 w_{12}\left[S_{1 / 2}\left(w_{1}\right)+T_{1 / 2}\left(w_{2}\right)\right]=0$ where $w_{12}\left[S_{1 / 2}\left(w_{1}\right)+T_{1 / 2}\left(w_{2}\right)\right]$ is the component of $w_{12}\left(w_{1}+w_{2}\right)$ in $\mathfrak{A}_{12}$. It is known [2, page 517; and 7] that $S_{1 / 2}\left(w_{1}\right)+T_{1 / 2}\left(w_{2}\right)$ is a nilpotent mapping. Since $w_{12} \neq 0$, it follows that $\gamma+\delta=0$, as desired.

3. Proof of Theorem 1. By hypothesis $\mathfrak{A}$ is nilstable with respect to $u$ and $f$, and, by Theorem $2, f=1 / 2(1+w), w=\gamma(u-v)+w_{12}+w_{1}$ $+w_{2}$. If $\gamma=0$, the proof of the theorem of reference [8] gives us the result of Theorem 1 . In making the induction of $\$ 4$ of [8], we consider only the class of algebras satisfying the hypotheses of Theorem 1. Thus we may now assume $\gamma \neq 0$. Even in this case the proof is patterned after that of reference [8]. Lemma 8 of [8] holds in our situation.

Next we proceed to derive a result comparable to Lemma 9 of [8]. Any element of $\mathfrak{A}_{f}(\lambda)$ has the form $a=\alpha u+\beta v+a_{12}+a_{1}+a_{2}$ where $\alpha, \beta$ are in $\mathfrak{F}, a_{1}, a_{2}$ are in $\mathfrak{B}=\mathfrak{S}_{1} \oplus \mathfrak{S}_{2}, a_{12}$ in $\mathfrak{A}_{12}$. Then $w a=\alpha \gamma u-\beta \gamma v$ $+\gamma\left(a_{1}-a_{2}\right)+1 / 2(\alpha+\beta) w_{12}+w_{12} a_{12}+w_{12}\left(a_{1}+a_{2}\right)+\alpha w_{1}+\beta w_{2}$ 
$+a_{12}\left(w_{1}+w_{2}\right)+a_{1} w_{1}+a_{2} w_{2}=(2 \lambda-1)\left(\alpha u+\beta v+a_{12}+a_{1}+a_{2}\right)$. If $\lambda=1$, Lemma 10 of [2] implies $w_{12} a_{12} \equiv \alpha(1-\gamma)=\beta(1+\gamma)$ where by $w_{12} a_{12} \equiv \alpha(1-\gamma)$ we mean $w_{12} a_{12}-\alpha(1-\gamma) 1$ is in S. From $\alpha(i-\gamma)$ $=\beta(1+\gamma)$ we get $2 \alpha=\alpha+\alpha \gamma+\beta(1+\gamma)=(\alpha+\beta)(1+\gamma)$ or $\alpha(1-\gamma)$ $=(\alpha+\beta)\left(1-\gamma^{2}\right) / 2 \equiv w_{12} a_{12}$. Also, $2^{-1}(\alpha+\beta) w_{12}+w_{12}\left[S_{1 / 2}\left(a_{1}\right)+T_{1 / 2}\left(a_{2}\right)\right]$ $+a_{12}\left[S_{1 / 2}\left(w_{1}\right)+T_{1 / 2}\left(w_{2}\right)\right]=a_{12}$. Multiply both sides by $w_{12} T_{1 / 2}\left(g_{2}\right)$, use the fact $w_{12}\left[S_{1 / 2}\left(w_{1}\right)+T_{1 / 2}\left(w_{2}\right)\right]=0$ which follows from our computation of $w^{2}=1$, and use the results of $[8, \S 2]$. Thus $a_{12} \cdot w_{12} T_{1 / 2}\left(g_{2}\right)$ is in (S) for any $g_{2}$ in $\mathcal{B}_{2}$. If $\lambda=0$, the calculations yield $w_{12} a_{12} \equiv-2^{-1}(\alpha+\beta)$ $\cdot\left(1-\gamma^{2}\right)=-\alpha(1+\gamma)=-\beta(1-\gamma)$. When $\lambda=1 / 2, w_{12} a_{12} \equiv-\alpha \gamma=\beta \gamma$ so $\gamma(\alpha+\beta)=0$ and since $\gamma \neq 0, \beta=-\alpha$. Some of these results are formally stated in the following lemma.

Lemma 1. Any element $a$ of $\mathfrak{A}$ may be written $a=\alpha u+\beta v+a_{12}+a_{1}+a_{2}$ with $\alpha, \beta$ in $\mathfrak{F}, a_{12}$ in $\mathfrak{A}_{12}, a_{1}, a_{2}$ in $\mathfrak{S}_{1}, \mathfrak{S}_{2}$, respectively. If $a$ is in $\mathfrak{A}_{f}(\lambda)$, $\lambda=0,1$, then $a_{12} \cdot w_{12} T_{1 / 2}\left(g_{2}\right)$ is in (S) for any $g_{2}$ in $\mathfrak{G}_{2}$. When $a$ is in $\mathfrak{A}_{f}(1 / 2), \beta=-\alpha$.

Now let $a$ be in $\mathfrak{A}_{f}(1)$ and let $b=\zeta u+\eta v+b_{12}+b_{1}+b_{2}$ also be in $\mathfrak{A}_{f}(1)$. Then $a b=\alpha \zeta u+\beta \eta v+2^{-1}(\alpha+\beta) b_{12}+2^{-1}(\zeta+\eta) a_{12}+a_{12} b_{12}$ $+a_{12}\left(b_{1}+b_{2}\right)+b_{12}\left(a_{1}+a_{2}\right)+\alpha b_{1}+\beta b_{2}+\zeta a_{1}+\eta a_{2}+a_{1} b_{1}+a_{2} b_{2}$ and $a b$ is in $\mathfrak{A}_{f}(1)$. Let $a b=\theta u+\phi v+c_{12}+c_{1}+c_{2}$ and let $a_{12} b_{12} \equiv \rho$. We have $\theta=\alpha \zeta+\rho, \phi=\beta \eta+\rho$. From the results above Lemma 1 , we have $(\alpha \zeta+\rho)(1-\gamma)=(\beta \eta+\rho)(1+\gamma)$. Also, $\quad \zeta(1-\gamma)=\eta(1+\gamma), \quad \beta(1+\gamma)$ $=\alpha(1-\gamma)$. Therefore, $\alpha \eta(1+\gamma)+\rho(1-\gamma)=\alpha \eta(1-\gamma)+\rho(1+\gamma)$, and and $2 \alpha \eta \gamma=2 \rho \gamma$. Since $\gamma \neq 0, \rho=\alpha \eta$ and $\theta=\alpha(\zeta+\eta), \phi=\eta(\alpha+\beta)$.

LeMmA 2. Let $a=\alpha u+\beta v+a_{12}+a_{1}+a_{2}$ and $b=\zeta u+\eta v+b_{12}+b_{1}+b_{2}$ be any two elements of $\mathfrak{A}_{f}(1)$. Then if $\gamma \neq 0, a_{12} b_{12} \equiv \alpha \eta$ and $a b=\alpha(\zeta+\eta) u$ $+\eta(\alpha+\beta) v+c_{12}+c_{1}+c_{2}$.

Corollary 1. Let $a=\alpha u+\beta v+a_{12}+a_{1}+a_{2}$ be any element of $\mathfrak{A}_{f}(1)$. Then $a^{k}=\alpha(\alpha+\beta)^{k-1} u+\beta(\alpha+\beta)^{k-1} v+c_{12}+c_{1}+c_{2}$.

Lemma 3. If $a$ is nilpotent, then $\alpha=\beta=0$.

The corollary to Lemma 2 implies that if $a$ is nilpotent $\alpha=0, \beta=0$ or $\alpha+\beta=0$. If $\beta=-\alpha$, the fact that $\alpha(1-\gamma)=\beta(1+\gamma)$ implies $\alpha=\beta=0$.

The results of Lemma 2, its corollary, and Lemma 3 can be proved in the same manner for elements of $\mathfrak{A}_{f}(0)$.

LEMMA 4. Let $a=\alpha u+\beta v+a_{12}+a_{1}+a_{2}$ be any element in $\mathfrak{A}_{f}(1)$ or $\mathfrak{A}_{f}(0)$ and $c=\delta(u-v)+c_{12}+c_{1}+c_{2}$ be any element of $\mathfrak{A}_{f}(1 / 2)$. Then $a_{12} c_{12} \equiv 2^{-1}(\beta-\alpha) \delta$. If $a$ is nilpotent, $a_{12} c_{12} \equiv 0$. 
The product $a c=m+n$ where $m$ is in $\mathfrak{A}_{f}(1 / 2)$ and $n$ is in $\mathfrak{A}_{f}(1-\lambda)$ when $a$ is in $\mathfrak{A}_{f}(\lambda),(\lambda=0,1)$. By assumption $\mathfrak{A}$ is nilstable with respect to $f$ so $n$ is nilpotent. Lemma 3 and the corresponding result for elements in $\mathfrak{A}_{f}(0)$ imply $n=n_{12}+n_{1}+n_{2}$ with $n_{1}, n_{2}$ in (3). By Lemma 1 , $m=\mu(u-v)+m_{12}+m_{1}+m_{2}$. From the equation $a c=m+n$ we obtain $\alpha \delta u-\beta \delta v+a_{12} c_{12} \equiv \mu(u-v)$ and it follows that $\mu-\alpha \delta=-\mu+\beta \delta$, $\mu=2^{-1}(\alpha+\beta) \delta, a_{12} c_{12} \equiv 2^{-1}(\beta-\alpha) \delta$.

As in [8], consider any element $g_{2}$ in $\left(5_{2}\right.$ and write $g_{2}=g_{f}(1)+g_{f}(0)$ $+g_{f}(1 / 2)$ where $g_{f}(\lambda)$ is in $\mathfrak{A}_{f}(\lambda)$. Write each $g_{f}(\lambda)$ as a sum of elements determined by the decomposition of $\mathfrak{A}$ relative to $u$. Let $g_{f}(1)$ $=\alpha u+\beta v+a_{12}+a_{1}+a_{2}, g_{f}(0)=\zeta u+\eta v+b_{12}+b_{1}+b_{2}, g_{f}(1 / 2)=\phi(u-v)$ $+d_{12}+d_{1}+d_{2}$. Then $\alpha+\zeta=-\phi=-\beta-\eta$. The results before Lemma 1 imply $\alpha(1-\gamma)=\beta(1+\gamma)$ and $\zeta(1+\gamma)=\eta(1-\gamma)$. Subtract the second from the first of these relations to get $\alpha-\zeta=\beta-\eta$. Add corresponding sides of this relation to $\alpha+\zeta=-\beta-\eta$ so that $\alpha=-\eta$, and then $\beta=-\zeta$. Now by Lemma 4 and for any $c_{12}$ belonging to $\mathfrak{I}_{f}(1 / 2)$, $\left(a_{12}-b_{12}\right) c_{12} \equiv 2^{-1}(\beta-\alpha) \delta-2^{-1}(\eta-\zeta) \delta=0$. By Lemma 8 of [8], $a_{12}-b_{12}=w_{12} T_{1 / 2}\left(g_{2}\right)$. Thus we have proved Lemma 12 and Theorem 1 of $[8]$.

The induction of $\$ 4$ of [8] made in the class of algebras nilstable with respect to two idempotents $u, f$ such that $u \neq 1, f \neq 1, u+f \neq 1$, $f \neq u+z_{12}+z_{1}+z_{2}$, and $f \neq v+z_{12}+z_{1}+z_{2}$ with $z_{12}$ in $\mathfrak{A}_{12}, z_{1}$ in $\mathfrak{A}_{1}, z_{2}$ in $\mathfrak{A}_{2}$, and the induction completes the proof of Theorem 1 . In order to successfully complete the induction it is necessary to have $w_{12}^{2}$ nonnilpotent. Since $w_{12}^{2} \equiv\left(1-\gamma^{2}\right)$, this means we need to have $\gamma^{2}-1 \neq 0$, $\gamma \neq \pm 1$. The condition $\gamma= \pm 1$ implies

$$
f=(1+w) / 2=u+2^{-1}\left(w_{12}+w_{1}+w_{2}\right)
$$

or $f=v+2^{-1}\left(w_{12}+w_{1}+w_{2}\right)$.

4. The cases $\gamma= \pm 1$. The result of Theorem 1 is not true when $\gamma= \pm 1$. For example consider the $u$-stable algebra $\subseteq$ of characteristic $p>5$ described in [6]. It is not a Jordan algebra and $\subseteq=u \mathfrak{A}+v \mathfrak{A}$ $+y_{0} \mathfrak{H}+y_{1} \mathfrak{A}$ where $\mathfrak{A}=\mathfrak{F}[1, x], x^{p}=0$. In the decomposition relative to $u, \Im_{12}=y_{0} \mathfrak{A}+y_{1} \mathfrak{A}, \mathfrak{S}_{1}=u \mathfrak{A}, \mathfrak{S}_{2}=v \mathfrak{A}$. Let $w=u-v+2 y_{1} x^{p-1}$ so that $f=2^{-1}(1+w)=u+y_{1} x^{p-1}$. If $a$ is in $\mathfrak{S}_{f}(1), a=\alpha u+\beta v+a_{12}+a_{1}+a_{2}$. The proof of Lemma 1 implies $\alpha(1-\gamma)=\beta(1+\gamma)$. Since $\gamma=1, \beta=0$. Furthermore $w a=a, \alpha u+a_{1}-a_{2}+\alpha y_{1} x^{p-1}+2 y_{1} x^{p-1} \cdot a_{12}=\alpha u+a_{12}+a_{1}$ $+a_{2}$ where we have used the fact that $y_{1} x^{p-1}\left(a_{1}+a_{2}\right)=0$. It follows that $a_{12}=\alpha y_{1} x^{p-1}$ and hence $a_{2}=0$. Thus $a=\alpha u+\alpha y_{1} x^{p-1}+a_{1}=\alpha f+a_{1}$ where $a_{1}$ is any nilpotent element of $\Im_{1}$. Similarly, if $b$ is any element of $\Xi_{f}(0), b=\beta v-\beta y_{1} x^{p-1}+b_{2}=\beta(1-f)+b_{2}$ where $b_{2}$ is any nilpotent 
element of $\mathfrak{S}_{2}$. Next let $c$ be any element of $\mathfrak{S}_{f}(1 / 2)$. By Lemma 1 , $c=\lambda(u-v)+c_{12}+c_{1}+c_{2}$ and $w c=0$. The product $w c=0$ implies $\lambda(u+v)+c_{1}-c_{2}+2 y_{1} x^{p-1} \cdot c_{12}=0$. The multiplication table of $\subseteq$ implies $2 y_{1} x^{p-1} \cdot c_{12}$ is nilpotent and therefore it is equal to $c_{2}-c_{1}$ and $\lambda=0$. The product $c a=\left(c_{12}+c_{1}+c_{2}\right)\left(\alpha f+a_{1}\right)=2^{-1} \alpha\left(c_{12}+c_{1}+c_{2}\right)+\left(c_{12}+c_{1}\right) a_{1}$. We know $c a$ is in $\Im_{f}(1 / 2)+\mathfrak{S}_{f}(0)$, so $w(c a)$ is the negative of the component in $\mathfrak{S}_{f}(0)$. Computing, we get $w(c a)=2 y_{1} x^{p-1} \cdot c_{12} a_{1}+c_{1} a_{1}$. By the definition of multiplication in $\subseteq, y_{1} x^{p-1} \cdot c_{12} a_{1}$ is 0 or a scalar multiple of $x^{p-1}$. Since $2 y_{1} x^{p-1} \cdot c_{12}=c_{2}-c_{1}, c_{1} a_{1}=-\left(2 y_{1} x^{p-1} \cdot c_{12}\right) a_{1}$, which is a scalar multiple of $x^{p-1} u$. Therefore, the component of $c a$ in $\mathfrak{S}_{f}(0)$ is nilpotent. Similarly, $c b$ is the sum of an element in $\mathfrak{S}_{f}(1 / 2)$ and a nilpotent element of $\mathfrak{S}_{f}(1)$. This proves that $\subseteq$ is nilstable with respect to $f$, and shows that the restriction $\gamma \neq \pm 1$ is necessary in order to obtain the result of Theorem 1. In our example we took $\gamma=1$, but we could just as easily let $\gamma=-1, w=v-u+2 y_{1} x^{p-1}$, and $f=v+y_{1} x^{p-1}$.

\section{REFERENCES}

1. A. A. Albert, Power-associative rings, Trans. Amer. Math. Soc. vol. 64 (1948) pp. 552-593.

2. - A theory of power-associative commulative algebras, Trans. Amer. Math. Soc. vol. 69 (1950) pp. 503-527.

3. - On commutative power-associative algebras of degree two, Trans. Amer. Math. Soc. vol. 74 (1953) pp. 323-343.

4. - On partially stable algebras, Trans. Amer. Math. Soc. vol. 84 (1957) pp. $430-443$.

5. - Addendum to the paper on partially stable algebras, Trans. Amer. Math. Soc. vol. 87 (1958) pp. 57-62.

6. L. A. Kokoris, Power-associative commutative algebras of degree two, Proc. Nat. Acad. Sci. U.S.A. vol. 38 (1952) pp. 534-537.

7. - New results on power-associative algebras, Trans. Amer. Math. Soc. vol. 77 (1954) pp. 363-373.

8. - Simple power-associative algebras of degree two, Ann. of Math. vol. 64 (1956) pp. 544-550.

WASHINGTON UNIVERSITY 